\title{
A Dynamically Reconfigurable Architecture for Emergency and Disaster Management in ITS
}

\author{
Smail Niar*, Arda Yurdakul ${ }^{\dagger}$, Osman Unsal ${ }^{\ddagger}$, Tuna Tugcu ${ }^{\dagger}$ and Aziz Yuceturk ${ }^{\S}$ \\ *University of Valenciennes, France, Email: smail.niar@univ-valenciennes.fr \\ ${ }^{\dagger}$ Bogazici University, Istanbul, Turkey, Email: \{yurdakul, tugcu\}@ boun.edu.tr

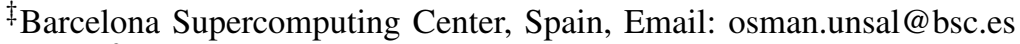 \\ §Vodafone, Turkey, Email: aziz.yuceturk@ vodafone.com
}

\begin{abstract}
Current transportation infrastructures are not designed to handle unexpected and undesired events or disasters. Emergency Vehicles (EV) such as ambulances, malfunctioning or broken cars, and accidents may appear at any time at any spot in the traffic. Less likely but not inevitably, there might be fires, terrorist attacks, earthquakes which will cause panic behaviour and unexpected traffic congestion. The challenge in our framework is the proposition of a range of innovative solutions for intelligent and efficient traffic management in Emergency and Disaster Management (EDM). In this paper, we propose a multilevel infrastructure which is dynamically reconfigured and thus allows a trade-off between total cost, energy consumption and quality of service in EDM.
\end{abstract}

Keywords-Dynamically reconfigurable systems, Distributed Communicating Infrastructure, V2X communications, incident management.

\section{INTRODUCTION}

Vehicle-to-Vehicle (V2V) and Vehicle-to-infrastructure (V2I) communications provide new opportunities to develop an unlimited range of applications that can make transport infrastructure management more efficient, provide advance in traffic safety and efficiency, and reduce fuel consumption. One of the biggest challenges is the exploitation and the analysis of the huge quantity of data that may be collected by existing in-vehicle or in-infrastructure devices (sensors, actuators, etc.).

In this work, our aim is to collect data that will be used to analyze and predict the behavior of traffic users and infrastructure capabilities for traffic evacuation or to send Emergency Vehicles (EV) such as ambulances or fire trucks to assist people. EV crashes and accidents in urban areas are more pronounced than in rural areas. Measurements done by Savolainen [9] shows that $82 \%$ of EV accidents happen in urban environments and most of them at intersections, particularly angle collisions at stop-controlled and signalized intersections. They noted that $91 \%$ of these crashes occurred while the vehicle was operating with lights and sirens activated. A common drawback with the current infrastructure types is that they are not designed to handle unexpected or undesired events and disasters. Ambulances, malfunctioning or broken cars, accidents may appear at any time at any spot in the traffic. Less likely but not inevitably, there might be fires, terrorist attacks, earthquakes which will cause panic behavior and unexpected traffic congestion.

Intelligent Transportation Systems (ITS) must take into account disaster situations such that the infrastructure is dynamically reconfigured according to the current traffic flux, traffic status, the road conditions, etc. To achieve this, different types of data from different sources are collected in real time. Most of these sources already reside on vehicle or at $\mathrm{road} /$ traffic observation points. Within the scope of this work, data from these resources will be aggregated, analyzed and fused in real time to automatically sense or estimate traffic congestion in real-time.

\section{StATE OF THE ART}

Software-based traffic monitoring applications such as Yandex Traffic, Google Traffic, Weza that operate on the internet/mobile telecommunication infrastructure fall short in providing true real-time data due to data travel time and response time. Usually the information is updated in more than five minutes. Another drawback is the lack of accuracy in the presented information. For example in Yandex Traffic, a road can be partitioned with green-red-green colors subsequently. When we try to read the velocity values, they can be $35 \mathrm{~km} / \mathrm{h}$ $3 \mathrm{~km} / \mathrm{h}-35 \mathrm{~km} / \mathrm{h}$. Weza is updated only by the users. So the update time can be as long as an hour. Hence, current software solutions are not real-time and the presented information is not accurate. As a result, they cannot be used in incident management.

In [2] [1], the authors proposes the Density-based Road Side Unit (D-RSU) deployment policy which consists on placing RSUs according to an inverse proportion to the expected density to reduce the deployment cost. At the opposite of our platform, the architecture is static. In addition, only the communication between the On-board unit (OBU) and the D-RSU is proposed in their approach. In our work, the communication and the processing capabilities of our multilayer architecture is adapted dynamically.

In [7], a Geospatial Information System (GIS) is used in conjunction with a relational database for a location based service and resource distribution in a EDM. The authors propose a distributed image processing algorithm to compute the disaster damage assessment map from satellite images. In our approach we use a different approach. Hundreds of connected processors and sensors embedded in the vehicle and the infrastructure in one side and communication tools in the other side, will be used to process, analyze, store and communicate a large quantity of data. This new feature will allow the definition of new services for Intelligent Transportation Systems (ITS) and the improvement of infrastructure capacity in EDM. 
The following table compares our work to exiting frameworks:

TABLE I. EXISTING FRAMEWORKS AND COMPARISON WITH OUR WORK

\begin{tabular}{|c|c|}
\hline Framework Name & Objectives \\
\hline & V2X communications \\
\hline CVIS & $\begin{array}{l}\text { Cooperative Vehicle-Infrastructure Systems that developed a } \\
\text { communication system capable to use various wireless tech- } \\
\text { nologies. }\end{array}$ \\
\hline iTETRIS & $\begin{array}{l}\text { Uses V2V-V2I communication technologies to improve traffic } \\
\text { management through real-time exchange of data among vehi- } \\
\text { cles and with road infrastructure }\end{array}$ \\
\hline CarTALK & $\begin{array}{l}\text { Advanced driver support system based on } \mathrm{V} 2 \mathrm{~V} \text { communication } \\
\text { technologies, development of cooperative driver assistance } \\
\text { systems and a self-organizing ad hoc radio network. A vehicle } \\
\text { sends a warning message when it detects a breakdown, high } \\
\text { traffic density, congestion or dangerous road surfaces. }\end{array}$ \\
\hline WiSafeCar & $\begin{array}{l}\text { The framework develops a reliable wireless traffic service } \\
\text { platform to improve traffic safety, avoid traffic accidents and } \\
\text { provide variety of new type of services to vehicles. }\end{array}$ \\
\hline DRIVE C2X & $\begin{array}{l}\text { Evaluate the impacts of given cooperative driving functions \& } \\
\text { services on driver behaviour and transport system. }\end{array}$ \\
\hline Our Work & $\begin{array}{l}\text { In comparison of the previous frameworks, our framework } \\
\text { does not focus only on communication between the different } \\
\text { layers of the infrastructure. Its aims also the development } \\
\text { of new HW and SW architectures. We will uses } 3 \text { types of } \\
\text { communications V2V, V2I and V2C. }\end{array}$ \\
\hline \multicolumn{2}{|r|}{ Optimizing Road Traffic and Congestion Reduction } \\
\hline SAFESPOT & Cooperative systems for road safety to prevent road accidents. \\
\hline INTERSAFE-2 & Development of the cooperative intersection safety systems. \\
\hline CarCoDe & $\begin{array}{l}\text { Develop a software platform which enables traffic service ICT } \\
\text { ecosystems and to offer a merging layer between automotive } \\
\text { industry, traffic service operators and third party developers. }\end{array}$ \\
\hline COMeSafety & $\begin{array}{l}\text { Communications for eSafety, the basis for cooperative intelli- } \\
\text { gent road transport systems. It provides an open integrating } \\
\text { platform, aiming for the interests of all public and private } \\
\text { Co-ordination and consolidation of research results and their } \\
\text { implementation. . }\end{array}$ \\
\hline COOPERS & $\begin{array}{l}\text { Cooperative Networks for Intelligent Road Safety, develop- } \\
\text { ment of innovative telematic applications on the road infras- } \\
\text { tructure, enhancement of road safety by direct and up-to-date } \\
\text { traffic information based on wireless communication between } \\
\text { infrastructure and motorized vehicles on a motorway section. }\end{array}$ \\
\hline Monaco & $\begin{array}{l}\text { A smart city initiative with mobile information services with } \\
\text { next-generation possible applications urban transport and mo- } \\
\text { bility. It monitors traffic, for example to act to give priority to } \\
\text { an ambulance at a red light. It can also control street lighting } \\
\text { depending on the presence or absence of pedestrians in the } \\
\text { streets and optimize energy. }\end{array}$ \\
\hline Our Work & $\begin{array}{l}\text { Optimization of road traffic is done in real-time using big } \\
\text { quantity of data coming from different types of transport data } \\
\text { providers: Radars, Lidars and traffic cameras in public and } \\
\text { private transport vehicles, from the CP. Flow of data from the } \\
\text { accident/explosion/earthquake location is continually captured } \\
\text { and analyzed even after the first alarm. }\end{array}$ \\
\hline \multicolumn{2}{|r|}{ Safety, Accident Assistant and Incident Management } \\
\hline eCall & $\begin{array}{l}\text { eCall is an European initiative (not a framework) to bring rapid } \\
\text { assistance to motorists involved in a collision anywhere in the } \\
\text { European Union. In case of a crash, the nearest emergency } \\
\text { center is called automatically or manually and a 'Minimum } \\
\text { Set of Data' is sent, which includes the exact location of the } \\
\text { crash site. }\end{array}$ \\
\hline Butler & $\begin{array}{l}\text { BUTLER aims at providing a horizontal platform that al- } \\
\text { lows building context-aware applications from various domains } \\
\text { (e.g., city, home, transport, healthcare) reusing heterogeneous } \\
\text { sensor/actuator networks. }\end{array}$ \\
\hline Our Work & $\begin{array}{l}\text { Our work is complementary to eCall and can use the eCall in- } \\
\text { frastructure. At the opposite of eCall and thanks to IDPs, com- } \\
\text { munication and data transfers are realized in permanence. Our } \\
\text { work is not limited to GSM, wireless V2X communications } \\
\text { are also used. We will reuse available relevant transportation } \\
\text { related enablers from the Butler framework. }\end{array}$ \\
\hline
\end{tabular}

\section{PROPOSED CONCEPTS AND APPROACHES}

\section{A. Platform Architecture}

The system architecture of the proposed platform is shown in Figure 1. The architecture consists of three layers:
- $\quad$ Sensor Processing (SP) layer: This layer corresponds to all possible real-time data providers in the transport system. By the term real-time data providers, we mean sensors:

- On the vehicles such as Radars, Lidars and Cameras,

- On the road, such as traffic surveillance cameras and different types of radars and/or vehicle detectors,

Various types of data can be collected. The data can be as raw as the speed information of the vehicle, or as complex as a number and types of cars and buildings after an explosion. SP will receive the commands from the Intelligent Data Processor (IDP) layer so as to act according to the surveillance decisions. SP layer is also in charge of communication with Human/Man Interface (HMI) for presenting the information from the local sensors or from the IDPs.

- Intelligent Data Processing (IDP) Layer: This layer corresponds to small but computationally powerful units that can manage the traffic in real-time in a diameter of about $1 \mathrm{~km}$. IDPs process the data for taking decisions for local routing, traffic flow control and incident management. IDP's achieve this by sending commands to the SP layer and to the neighboring IDPs. IDPs will also filter the input data in such a way that only the data that will be used in global decision taking will be transferred to the CP. It should be noted that IDPs may preprocess the filtered data so that the amount of the data transferred to the cloud is significantly reduced. IDPs receive also data from the Cloud processing layer so as to reflect the global traffic management decisions to the neighboring IDPs and the SPs.

- Cloud Processing (CP) layer: This layer is composed of different Cloud servers. The servers are located at different parts of the city so that the risk of complete infrastructure failure is minimized. Cloud servers are used for taking global actions in the infrastructure such as global traffic management decisions and surveillance systems. This layer has its real-time inputs only from the IDP layer. CP fuses real-time data with the static data so as to service not only vehicles but also to people travelling in the city. Real-time data can be also archived for future/dynamic planning of public transport services.

Figure 2 gives a deployment of our plate-form in an explosion scenario.

Our infrastructure is in accordance with the IEEE 802.11p wireless access in vehicular environments (WAVE) standard. In WAVE, Roadside units (RSUs), only collect vehicular data. However, IDPs collect not only the vehicular data but also data from data provided from on-road sensors, public transport vehicles and local authorities like traffic centers, transport coordination units of cities, towns. Hence, they will be installed not on roads but also at public transport stations. The main advantages of cellular networks are their availability and coverage, but this kind of network is centralized which quickly becomes unusable when one of the central elements 


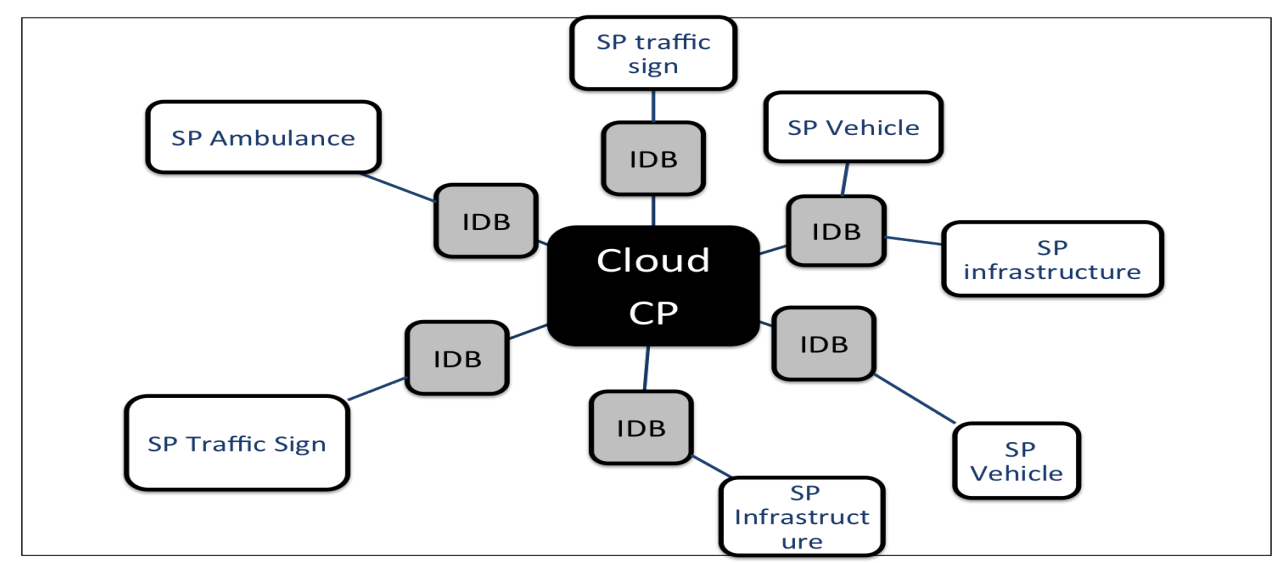

Fig. 1. The multi-layer infrastructure architecture

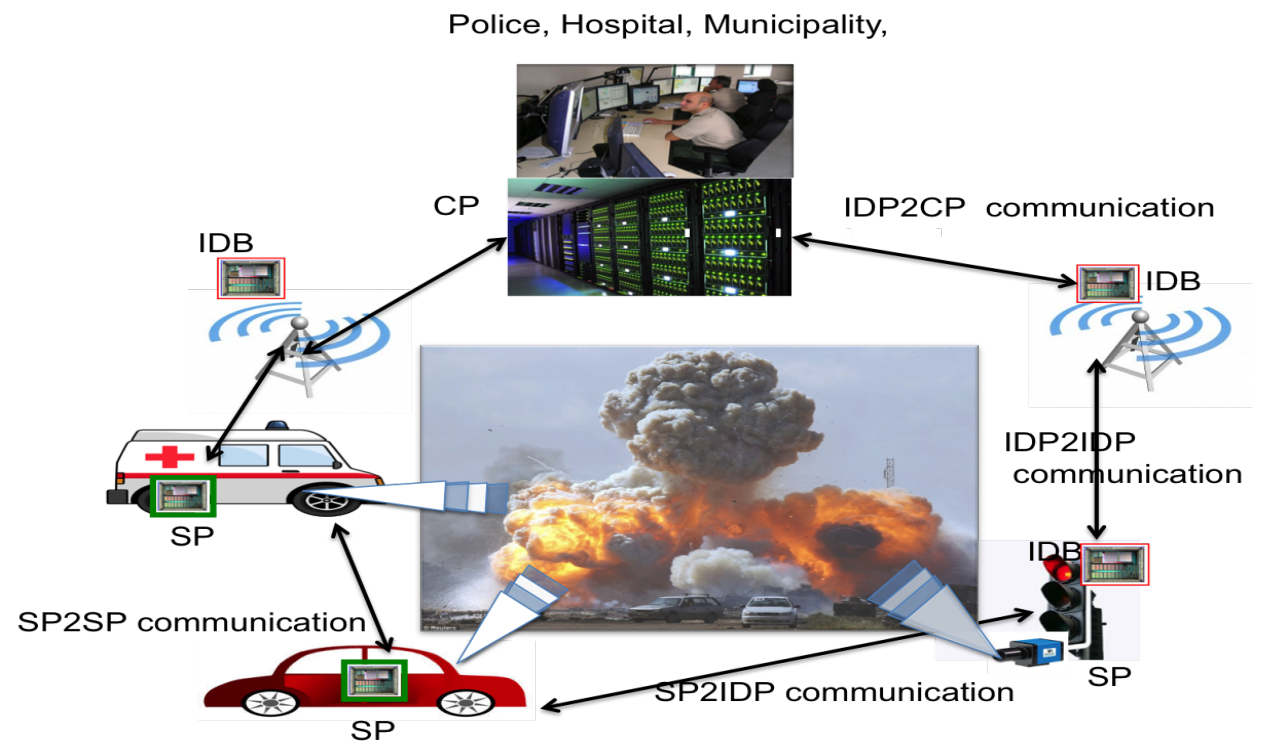

Fig. 2. The multi-layer infrastructure utilisation in EDM

is affected by a disaster.

Just like RSUs in WAVE, IDP layer demonstrates a selforganizational behavior. In other words, if some IDPs fail or a CP fails, the remaining IDPs will connect with each other and continue servicing. If a cluster of IDPs goes off, then the remaining IDPs will communicate over the $\mathrm{CP}$ layer if the communication range between IDPs is too long. Otherwise they will continue servicing locally until the necessary links are provided. Hence, due to its distributed and self-organizational nature, the infrastructure is resilient to malicious acts and to failures. If desired, an IDP can be installed and introduced to the infrastructure in an ad-hoc manner. IDP layer can be regarded as the local traffic management unit that will be effective in a cell with about $1 \mathrm{~km}$ diameter. Yet, for taking an immediate action that will affect a wide area, Cloud will be used, for example for routing the vehicles in an evacuating operation in case of floods or earthquakes. For example, if an ambulance wants to reach the most suitable hospital which is $10 \mathrm{~km}$ away, then the CP will query the IDPs on the region between these two points. The $\mathrm{CP}$ will also receive information, process, and send the fastest and secured route to the ambulance by utilizing the IDP situated in the road. In the mean time, it may also send directions to the traffic signs on the lanes so that one lane will be evacuated for the travel of the ambulance.

\section{B. SP, IDP and CP implementation}

SP nodes will bring computation to the data at the first level, filtering various types of data at different speeds, for local and global traffic management. For example, cameras provide images and video, but loop detectors provide speed and velocity data. The data rate also varies between different sensor types. The vehicle transmits its data at $27 \mathrm{Mbps}$ via $802.11 \mathrm{p}$, the data rate for a city camera is $500 \mathrm{Mbps}$, but on the other hand, only 64bps need to be transferred for an icy road detector.

Processing data for local traffic management, packing data for global data processing, sending/receiving data to/from different layers in real time falls in the domain of big-data 
and stream processing. For this purpose, machine-learning algorithms, stream processing architectures and methods for big data analytics are exploited. IDPs have to be small, low-cost and energy efficient. This brings the challenge of designing an embedded big-data/stream-data processing platform. Multicore embedded processing platforms have already been proposed by several microprocessor vendors, like ARM or Infineon. Yet, these platforms have not been designed for streaming big data, which requires efficient management/acceleration of embedded database. Field Programmable Gate Arrays (FPGAs) are widely used in accelerator design or prototyping. Moreover, with the dynamic reconfiguration property of the FPGAs, a low energy profile can be achieved in an embedded system that realizes multiple applications in a time-multiplexed manner. Hence, a programmable heterogeneous Multi-Processor System-on-Chip (MPSoC) that contains an FPGA with multiple processing cores is used here to appropriately deal with the requirements of real-time streaming computations on big data.

If the IEEE $802.11 \mathrm{p}$ manages a summary priority among users and dedicate special channels for priority communications, cellular networks manage different users with the same priority and quickly saturates face increased network load especially if severe. We offer to develop a cross-layer and cross-technology approach to build a scalable and configurable network, to ensure a minimum service and enhance the QoE. The proposed network architecture will be able to enhance the availability of the network especially during the golden hour .

Figure 3 gives the architecture of the IDP unit. This unit may contain different communication tools like IEEE 802.11p and LTE. A dynamically reconfigurable FPGA-based unit attached to a programmable multi-cores architecture is used. Depending on the application, the reconfigurable unit can be programmed to implement different intensive image and signal processing tasks.

\section{PlatForm FEATURES FOR EMERGENCY AND DISASTER MANAGEMENT}

Golden hour is known as the time between the occurrence of an incident such as traffic accident, car crash, hitting a pedestrian, and the intervention by a specialized trauma team [8]. If the casualties reach the operating table more than 60 minutes, the survival chance falls sharply. Studies show that only $30 \%$ of road accident victims in developed countries, and only $10 \%$ in developing countries, receive the adequate care in hospitals during the golden hour. We think that for natural disasters the situation is even more catastrophic.

Getting immediate information about the accident or the disaster and rapid acquiring of details such as the type of injuries and location cuts down emergency services' response time by $50 \%$, especially in congested urban areas.

For this reason, functionalities and services proposed by our platform are useful in Emergency and Disaster Management (EDM). This concerns in particular incident management by means of a dynamically reconfigured multi-layer distributed data infrastructure. According to the current traffic flux, the traffic status or the road and weather conditions, the HW and the SW are modified in such a way to implement new services in real-time. The utilization of a dynamically reconfigurable system to propose useful and efficient services in EDM is challenging. However, this approach raises important issues which need to be addressed. Effectiveness, reliability and robustness of the system are among the most important problems.

Our platform allows processing and exploitation in realtime of the big quantity of data coming from the different sensors and cloud centers. Municipalities, transportation department and companies, police, fire department etc. may take profit of the proposed platform as this will reduce the time of emergency arrival on the accident or natural disaster location, the evacuation of vehicles and people, and take care of the injuries.

The detection time of the accident or natural disaster and the automatic acquisition of accurate information on the corresponding location and conditions will be obtained in few seconds. Rapid processing of these data by our 3 layers will also allow determining secured shortest roads to be taken by emergency vehicles to the disaster location and for evacuation.

The proposed platform can also be used to record data from the different sensors in order to post-analyse undesired events such as explosion, earthquake that impact transportation infrastructures. By analyzing the big quantity of data that are collected from a large amount of sensors, the authorities will be able to optimize the use of the existing transportation infrastructure in EV routing and emergency evacuation.

In this section, we present the manner by which the infrastructure can be used in EDM. The main services and features of the platform will be presented in the following:

\section{A. Data Capturing}

The performance of our platform and its efficiency depend mainly on the number of sensors that are close to the incident or the disaster location. In this framework, we assume that the number and types of sensors in next vehicle generations will be more and more important. Thus, the problem is the efficient exploitation of the data sent by the sensors to offer useful services.

Here data are captured using multiple sensors: Camera, Radar, Lidar, GPS. The use of different types of sensors allows to have complementarity between information sent by the sensors on the details of the disaster and also tolerate failures in the sensors in EDM. The data to be sent from these sensors to the SP then to IDP then to CP and the corresponding bandwidth are controlled dynamically function of the emergency layer of the area where the SP, i.e. the vehicle, or the IDP are situated. More the vehicle or the IDP are closer to the disaster location, more the corresponding SPs and IDPs are allowed to send important quantity of data to close IDPs. Once the incident is detected, all the sensors in the area of the incident should be configured to achieve effective emergency duties.

\section{B. Processing}

At the SP and IDP layers, a high quantity of low level processing operations must delivered in real time. The type and localization of the operation to be implemented at the 3 layers. Image and signal processing functions such as filtering, merging and fusion, partitioning, pattern matching, and 


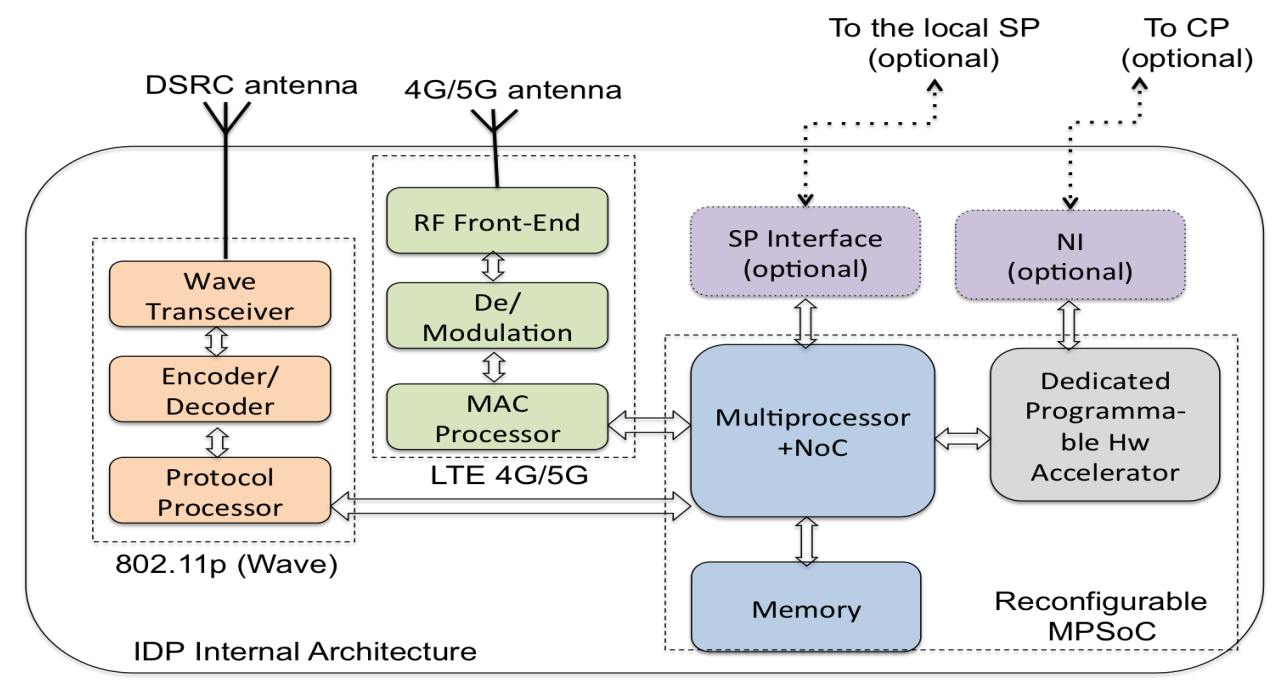

Fig. 3. IDP internal architecture

data encryption/decryption functions are among the possible operators that will be implemented in the SP and IDP layers (figure 4).

In [5], it was demonstrated that the utilization of shortrange radar to detect and identify 20 obstacles requires a processing power of 4 GFLOPS. When multiple sensors, such as long and short, front and rear radars, Lidars and cameras, must be used, the SP must provide a significantly increased processing power. Embedded multicore architectures in form of Multi-Processor System-on-Chip (MPSoC) and FPGAbased dynamically reconfigurable embedded systems could be an efficient solution to provide high processing bandwidth [6].

At the IDP and SP layers, big data processing, such large data-base design, and stream data processing need high performance computing and communication bandwidth. In this framework, basic processing operators are mapped on dynamically FPGA-based reconfigurable accelerators, embedded multiprocessors and libraries for sensing and multimedia applications.

One of the most important feature in this work is that preprocessing at the infrastructure at SP and IDP layers, makes detection of complex events possible. In addition, this preprocessing will reduce execution time at IDP and CP and comply with real-time constraints of EDM applications, such as golden hour. Most of the existing work in EDM for ITS, do not consider data sensing and data fusion from multiple types of sensors, vehicles and infrastructure. For example in [3], the authors propose a GPU-FPGA-CPU platform for data fusion as an RSU. The target application is pedestrian detection using 2 cameras, one for visible range and the other for far infrared range. The authors do not consider data from on-the-vehicle sensors.

\section{Communication}

EDM applications have special needs in communications. In our approach of EDM for ITS, a large quantity of raw data must be transmitted between the different nodes at the different layers. In addition different communication interfaces for different sensor types are used. For SP2IDP communication, its difficult to transmit data at a high data-rate in the case of high mobility with LTE and IEEE.802.11p solutions. In addition, to transfer intelligent data, a decision method obtained by system modelling to reduce the amount of raw data that needs to be transferred. High mobility, multimodes communication with LTE/4G/5G and 802.11p are among the most important challenges for the communication functionality of the platform. Interfacing all sensors at SP and IDP layers with wireless 802.11 p or $3 \mathrm{G} / 4 \mathrm{G}$ technologies are is also provided.

\section{Data storing}

Thanks to its large number of nodes and layers, a large memory to hold various types of real-time and static data is provided by the proposed platform. When an SP moves from one IDP area to another, data coherency problem appears for the data stored in the different IDP. To solve this problem, the different memory units are considered as a unique hierarchical memory with 2 layers: IDP and CP.

\section{E. Reliability}

Our distributed and heterogeneous system with its 3 layers can make the system very complex, vulnerable and fragile. In most of the exiting solutions, reliability is ensured with complex and costly solutions. An EDM platform must work even if one of more components has failure. Due to its distributed and self-organizational nature, our infrastructure will be prone to malicious acts. In order to recover from failures, the system has the capability to detect failure and make decision, performing its required functions under stated conditions for a specific period of time.

Using SP2X communication, when a local sensor breaks it is possible to use data from the IDP. In addition, disturbances and uncertainties, the SP is enhanced by new virtual sensors. At the cost of a reduced additional processing and communication overheads, virtual sensors can increase system robustness and reliability. When a sensor becomes defective due to ageing 


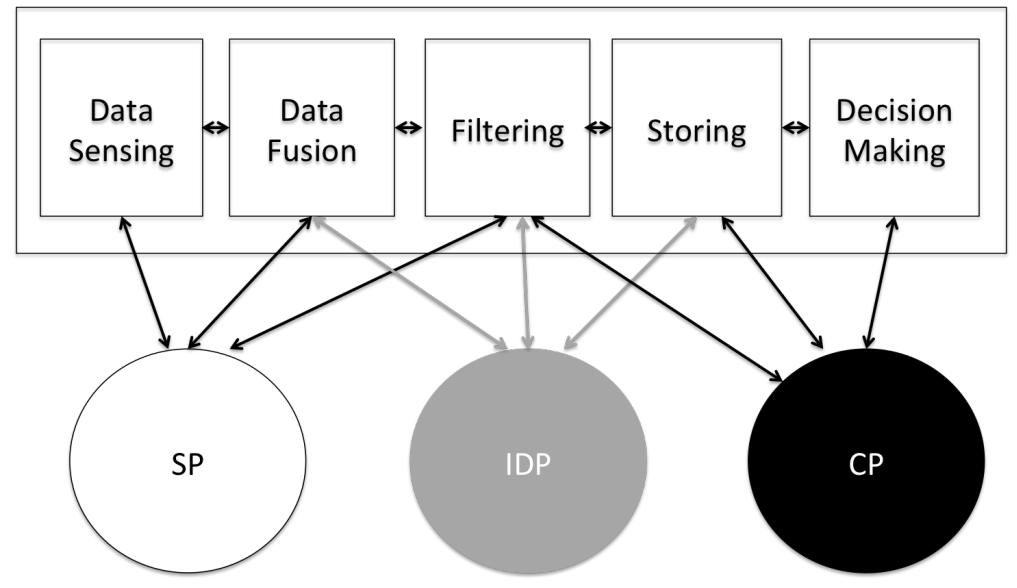

Fig. 4. EDM application decomposition in tasks and their mapping to the different platform layers

for instance, it is removed and replaced by a virtual sensor that uses data from other sensors, from the IDPs or from the CP.

In addition, it will be easy to introduce new IDPs after deployment of the infrastructure. Even if some IDPs fail, other IDPs and the Cloud infrastructure will continue to function.

\section{F. Security}

High level of security must be provided with a reduced cost and reduced energy consumption. IDP2IDP and IDP2CP interfaces will have network security provided by mobile telecom operator. UEA0/UEA1, UMTS encryption algorithms are used in UMTS (3G) networks. Thus the communication subsystem is assumed to be robust for threats and attacks.

For V2V and V2I communications, the usage of $802.11 \mathrm{p}$ will be exploited to acts as a gatekeeper for basic network access. IDPs will support the IPSec suite of standards. If IDP has no such kind of support, then external devices collocated with the IDP can provide IPSec transport/tunneling.

\section{CONCLUSION AND FUTUR WORKS}

In this paper, we presented a cooperative research project that aims improving the transportation infrastructure capacity and safety. This concerns in particular EDM by means of a dynamically reconfigured multi-layer distributed data infrastructure. According to the current traffic flux, traffic status, the road and the position of the IDP or the SP, the corresponding communication and processing tools are modified in such a way to implement in real-time the desired services. This allows processing and exploitation in real-time of a big quantity of data coming from the different sensors and cloud centers.

Municipalities, transportation department and companies, police, fire department etc. will take profit of the proposed platform as it will greatly reduce the time of emergency arrival on the accident or natural disaster location, the evacuation of vehicles and people, and take care of the injuries. The impact is reduced to a few seconds, the detection time of the accident or natural disaster and the automatic acquisition of accurate information on accident/disaster location, conditions, etc. Rapid processing of these data will also allow determining secured shortest roads to be taken by emergency vehicles to the disaster location.

\section{ACKNOWLEDGEMENTS}

The authors acknowledge support given by the Scientific and Technological Research Council of Turkey (TB?TAK) to Prof. S. NIAR under Project No: 2221 and by the Railenium Institute of Technological Research (IRT) France.

\section{REFERENCES}

[1] J. Barrachina, P. Garrido, M. Fogue, and F.J. Martinez. D-rsu: A densitybased approach for road side unit deployment in urban scenarios. In IEEE Intelligent Vehicles Symposium, June 2012.

[2] J. Barrachina, P. Garrido, M. Fogue, F.J. Martinez, J.-C. Cano, C.T. Calafate, and P. Manzoni. Road side unit deployment: A density-based approach. Intelligent Transportation Systems Magazine, IEEE, 5(3):3039, Fall 2013.

[3] S. Bauer, S. Kohler, K. Doll, and U. Brunsmann. Fpga-gpu architecture for kernel svm pedestrian detection. In Computer Vision and Pattern Recognition Workshops (CVPRW), 2010 IEEE Computer Society Conference on, 2010.

[4] Bharat Dogra. Save the golden hour. http://www.thehindu.com/features/ metroplus/society/save-the-golden-hour/article5268987.ece, 2013.

[5] H. Liu Haisheng and S. Niar. Radar signature in multiple target tracking system for driver assistant application. In Design, Automation Test in Europe Conference Exhibition (DATE), March 2013.

[6] N. Harb, S. Niar, and M. Saghir. Dynamically reconfigurable embedded architectures for safe transportations systems. In A. Bagnato \& Al., editor, ndustrial and Research Perspectives on Embedded System Design. IGI GLOBAL, 2014.

[7] Ge Jin, Barbara Nicolai, Keyuan Jiang, and Charles Winer. Distributed image processing and classification for gis based disaster management and communication system. In Proceedings of the $2 N d$ International Conference on Computing for Geospatial Research \&Amp; Applications, COM.Geo '11, pages 27:1-27:6, 2011.

[8] F.J. Martinez, Chai-Keong Toh, J.-C. Cano, C.T. Calafate, and P. Manzoni. Emergency services in future intelligent transportation systems based on vehicular communication networks. Intelligent Transportation Systems Magazine, IEEE, 2(2):6-20, Summer 2010.

[9] P.T. Savolainen. Investigation of emergency vehicle crashes in the state of michigan,. INEXTRANS project, (015WY01), 2013. 